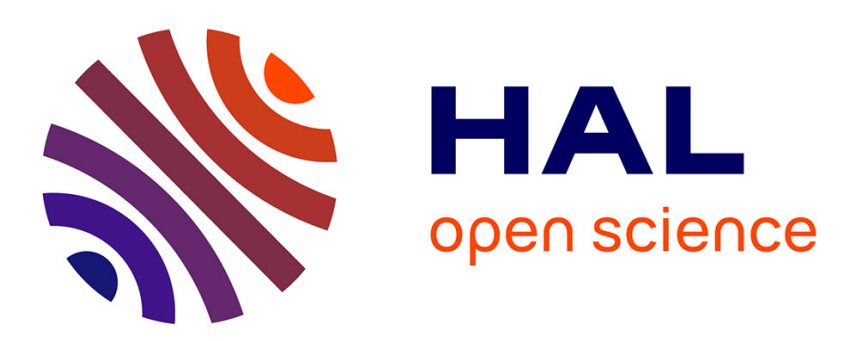

\title{
Les scholastiques dans la correspondance patriarcale de Sévère d'Antioche (512-518)
}

Frédéric Alpi

\section{To cite this version:}

Frédéric Alpi. Les scholastiques dans la correspondance patriarcale de Sévère d'Antioche (512-518).

Topoi Orient Occident. Supplément, 2007. hal-03317907

\section{HAL Id: hal-03317907 \\ https://hal.science/hal-03317907}

Submitted on 8 Aug 2021

HAL is a multi-disciplinary open access archive for the deposit and dissemination of scientific research documents, whether they are published or not. The documents may come from teaching and research institutions in France or abroad, or from public or private research centers.
L'archive ouverte pluridisciplinaire HAL, est destinée au dépôt et à la diffusion de documents scientifiques de niveau recherche, publiés ou non, émanant des établissements d'enseignement et de recherche français ou étrangers, des laboratoires publics ou privés. 


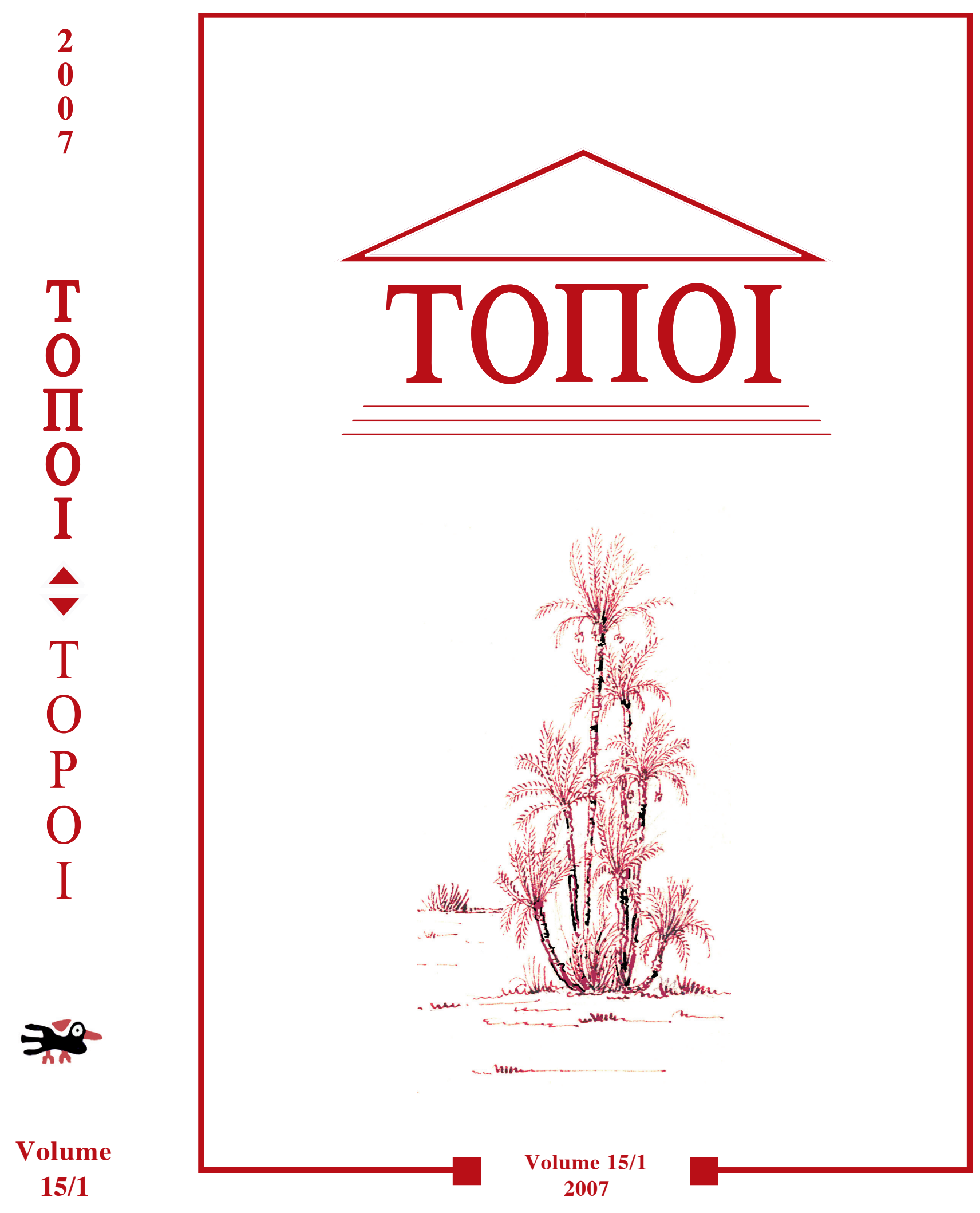




\section{SOMMAIRE}

\section{Fascicule 1}

Sommaire $\quad 3$

Index des auteurs $\quad 5-6$

\section{Dossiers et chroniques}

\section{Recherches sur la Babylonie hellénisée}

T. Bory, « Assyriology and the history of the Hellenistic period»

$\mathrm{Ph}$. Clancier, « La Babylonie hellénistique. Aperçu d'histoire politique et culturelle»

\section{Synthèse sur les plantes}

S. Amigues, «L'exploitation du monde végétal en Grèce classique et hellénistique. Essai de synthèse »

\section{La chronologie thessalienne}

B. Helly, « La capitale de la Thessalie face aux dangers de la troisième guerre de Macédoine : l'année 171 av. J.-C. à Larisa »

R. Bouchon, «Les "porteurs de toge" de Larissa »

\section{Temples et sanctuaires du Proche-Orient antique}

L. TholBecQ, « Hérodiens, Nabatéens et Lagides dans le Hawrān au $\mathrm{I}^{\mathrm{er}}$ S. av. J.-C. : réflexions autour du sanctuaire de $\mathrm{Ba}^{\circ}$ alšamīn de $\mathrm{Si}^{`}$ (Syrie du Sud) »

Les élites urbaines dans l'Empire romain d'Orient (IV -VI siècles)

(Dossier coordonné par V. Puech)

V. PUECH, «Introduction»

B. CABouret, « Les élites urbaines d'Antioche et de Syrie du Nord au Iv siècle » 319-341

S. MÉtivier et S. Destephen, «Chorévêques et évêques en Asie Mineure au IV ${ }^{e}$ et $V^{e}$ siècles »

V. Puech, «Élites urbaines et élites impériales sous Zénon (474-491) et Anastase (474-518)»

F. ALPI, «Les scholastiques dans la correspondance patriarcale de Sévère d'Antioche (512-518) »

K. MerLIN, «Venantius et Italica de Syracuse, notables siciliens de la fin du vi siècle » 


\section{LES SCHOLASTIQUES \\ DANS LA CORRESPONDANCE PATRIARCALE DE SÉVÈRE D'ANTIOCHE (512-518) ${ }^{1}$}

On peut attendre des progrès des recherches en prosopographie tardo-antique une meilleure définition des fonctions sociales et politiques exercées par les membres de groupes professionnels urbains dont les contours restent encore imprécis ou le statut mal défini. À la marge des élites municipales de l'empire proto-byzantin, récemment étudiées par A. Laniado ${ }^{2}$, j'aborderai ici une catégorie particulière, celle des skholastikoi, telle que la documente, au début du $\mathrm{vI}^{\mathrm{e}} \mathrm{s}$., la correspondance du patriarche Sévère d'Antioche. Au contraire des curiales, détenteurs au premier chef d'une fortune foncière (ktètores), et dont l'autorité paraît aller décroissant, ceux-là tirent revenus et position de la pratique d'une profession intellectuelle dont le prestige se renforce avec le temps. Le mot lui-même a connu une évolution sémantique qui semble parallèle à la promotion sociale des personnels désignés ${ }^{3}$. À l'époque romaine, une telle appellation peut s'appliquer aux membres de l'élite, qui ont accès à la culture grecque pour avoir fréquenté la schola du rhetor ${ }^{4}$. De fait, cette première acception semble se maintenir assez tard comme épithète

1. Je présente ici ma communication à la journée d'étude sur « Les élites urbaines dans l'Empire romain d'Orient $\left(\mathrm{IV}^{\mathrm{e}}-\mathrm{v} \mathrm{I}^{\mathrm{e}} \mathrm{s}\right.$.) », organisée le 11 juin 2004, à l'Université de Versailles-Saint-Quentin-en-Yvelines, par le laboratoire URMED (UMR 8140). Je remercie Vincent Puech de m'y avoir convié, ainsi que tous les participants pour la discussion féconde qui s'ensuivit. Ma reconnaissance s'adresse aussi à Pierre-Paul Corsetti, qui a relu mon texte, et à la rédaction de Topoi qui a bien voulu accueillir cette étude.

2. LANIADO 2002.

3. Preisigke 1921 ; Claus 1965.

4. Marrou 1965, p. 413.

Topoi 15 (2007)

p. $397-411$ 
apposée au nom de personnages distingués ${ }^{5}$. Du $\mathrm{IV}^{\mathrm{e}}$ au $\mathrm{v}^{\mathrm{e}} \mathrm{s}$., cependant, à mesure que les juristes gagnent en influence, c'est l'École de droit qui devient, dans l'opinion lettrée, l'établissement d'enseignement supérieur par excellence, et le titre de skholastikos s'attache alors de préférence à des praticiens des lois, formés à Constantinople, à Alexandrie et surtout à Béryte ${ }^{6}$. Également qualifiés de la sorte, Zacharie de Mytilène $(\approx 465-550)^{7}$, Évagre d'Épiphanie $(\approx 536-594)^{8}$ ou Jean III ${ }^{9}$, patriarche de Constantinople de 565 à 577 , en portent témoignage. Le sens de skholastikos paraît toutefois moins précis que l'un des termes retenus pour désigner un «avocat plaidant»: causidicus, en latin; dikanikos ou synègoros, en grec ; dikaniko, dans les sources syriaques. On y verrait assez volontiers un équivalent tardo-antique de l'anglais lawyer, tout en relevant qu'avec la montée en puissance des techniciens du droit dans l'appareil de l'État et parmi les classes supérieures, cette qualification finit par s'attacher à de nombreux dignitaires ${ }^{10}$. Le dossier des skholastikoi présents dans la correspondance sévérienne comme destinataires d'épîtres patriarcales ou simplement mentionnés dans des lettres du prélat offre l'occasion de faire le point, à un moment précis de l'histoire de l'Église d'Antioche et de l'Empire d'Orient, sur le rôle, le statut et l'influence de ces spécialistes.

5. C'est toujours en ce sens qu'il faut comprendre le titre donné à l'historien Socrate de Constantinople ( $\approx 380-440)$. Cf. Maraval 2004, p. 10-11.

6. Collinet 1925, p. 29 (n. 2), 32, 46, 104.

7. PLRE 2, p. 1194-1195 : Zacharias 4 ; PChBE 3, p. 960-973 : Zacharias 1. Originaire de Gaza et auteur d'une Vita Seueri (CPG 6999) qui décrit en détail la vie de ses condisciples de Béryte, dont celle de son ami Sévère, Zacharie le Scholastique s'établit à Constantinople à l'issue de ses études (492). Après 518, son ralliement à l'orthodoxie chalcédonienne lui vaudra le siège épiscopal de Mytilène.

8. Historien le plus notable de la controverse chalcédonienne dans son Historia ecclesiastica (CPG 7500), il exerça comme skholastikos à Antioche, où il devint un proche conseiller du patriarche Grégoire (570-593).

9. Skholastikos antiochien élevé sur le trône patriarcal de la ville impériale. On lui attribue la composition du premier recueil systématique de droit ecclésiastique, la Synagôgè kanonôn (CPG 7550), et une compilation des Novelles de Justinien relatives aux affaires de l'Église (CPG 7551).

10. Qui peuvent d'ailleurs appartenir à l'élite traditionnelle des notables locaux. Cf. Laniado 2002, p. 85, 142, 151, 158, 179, 213, 218. 


\section{Le dossier}

Principalement rassemblée en deux collections syriaques ${ }^{11}$, la vaste correspondance de Sévère s'inscrit dans l'action juridictionnelle et la politique partisane de cet adversaire résolu de la christologie chalcédonienne, devenu en 512, dans le cadre de l'Hénotique et par la faveur d'Anastase, patriarche d'Antioche, siège dont il demeura le titulaire effectif jusqu'à la mort de cet empereur (été 518) ${ }^{12}$. Parmi ses correspondants directs, on peut alors compter une demi-douzaine de skholastikoi, corporation que Sévère honore ordinairement de l'appellation laudative de skoulstiqe m'lile (éloquents scholastiques). Il écrit ainsi à un skholastikos de Bosra nommé Aurélios ${ }^{13}$, en réponse à une question de ce dernier sur la possibilité canonique de maintenir en fonction un prêtre eunuque ${ }^{14}$. Cette lettre double d'ailleurs une missive adressée, dans les mêmes termes, au métropolite Cassien ${ }^{15}$, évêque du lieu ${ }^{16}$, auprès duquel Aurélios jouait apparemment le rôle de conseiller juridique. Entre 518 et 538, après son éviction consécutive à l'avènement de Justin $\mathrm{I}^{\mathrm{er}}$, mais alors qu'il se considérait toujours comme le patriarche légitime, Sévère écrit à un autre skholastikos de Bosra, Jean ${ }^{17}$, sur la question des castrations volontaires chez les clercs et les laïcs ${ }^{18}$.

11. The sixth book of the select letters of Severus, patriarch of Antioch $=S L(C P G 7070$ [1]); A collection of letters of Severus of Antioch $=C L$ (CPG 7070 [2]). Constitué au $\mathrm{VII}^{\mathrm{e}} \mathrm{s}$., en milieu chrétien jacobite et afin de disposer de textes normatifs, le premier recueil rassemble des lettres ou des fragments de lettres traitant de cas disciplinaires. Le second résulte d'une collation moderne de manuscrits à caractère plutôt théologique ou exégétique. Il existe encore un certain nombre de fragments inclus dans les chaînes ou les florilèges, voire dans des actes conciliaires chalcédoniens, à titre de testimonia haeretici (CPG 7071, 7080). On a pu reconnaître enfin d'autres lettres éparses (CPG 7070 [3-16]). Nous ne retenons ici, pour l'essentiel, que des missives dont l'éditeur moderne aura pu rapporter la rédaction aux années du patriarcat.

12. Anastase meurt dans la nuit du 8 au 9 juillet, le chalcédonien Justin $\mathrm{I}^{\mathrm{er}}$ lui succède aussitôt et Sévère doit quitter Antioche en septembre.

13. PLRE 2, p. 201 : Aurelius 2. Bosra, métropole de la province d'Arabie, relevait du ressort d'Antioche.

14. SL 8, 3, p. 443-445 [395-396].

15. $S L$ 8, 2, p. $442-443$ [394-395].

16. Vers 512-515, il remplace le chalcédonien Julien, qui se réfugie alors en Palestine et recouvrera son siège en 518, après la chute de Sévère. Cf. Honigmann 1951, p. 7677.

17. PLRE 2, p. 609 : Iohannes 66.

18. SL 8, 4, p. 460-466 [408-412]. 
Dans les deux cas, il fonde sa réponse sur la législation de Nicée (325), canon 1 (censure des mutilations délibérées; acceptation des opérations chirurgicales et des séquelles de maladies) ${ }^{19}$. Le skholastikos apparaît ici comme un responsable important de l'administration épiscopale et sa spécialité disciplinaire se vérifie dans le classement que le compilateur jacobite aura plus tard adopté, en plaçant les trois fragments à la suite, dans la même section du recueil normatif de lettres sévériennes $(S L 8)^{20}$. De plus, il convient de noter que Jean de Bosra se trouve, par ailleurs, le destinataire d'une explication théologique de Sévère sur la parousie, signe qu'il se situait bien dans la communion du patriarche ${ }^{21}$.

Ces deux qualités de compétence technique et de sympathie doctrinale se trouvent réunies au plus haut point, toujours dans le ressort d'Antioche, chez le principal soutien de Sévère dans la difficile province d'Isaurie, le comte Oecuménios ${ }^{22}$. C'est le diacre Libératus de Carthage qui nous apprend, dans sa relation des querelles christologiques des $\mathrm{V}^{\mathrm{e}}-\mathrm{VI}^{\mathrm{e}} \mathrm{s}$., que ce notable titré exerçait également les fonctions de skholastikos ${ }^{23}$. La correspondance sévérienne indique qu'il se trouvait en relation avec le futur patriarche dès les années du séjour que celui-ci fit à Constantinople (508-511), alors qu'il gagnait la confiance d'Anastase et nouait, depuis la cour, des contacts en province, notamment avec le métropolite Constantin de Séleucie ${ }^{24}$. Une fois devenu patriarche, Sévère prend appui sur cet ami de plume et le désigne sans ambages au successeur de Constantin, Solon de Séleucie, comme son fondé de pouvoir en Isaurie, proposant au nouveau métropolite de s'en référer à l'expertise d'Oecuménios pour régler les cas disciplinaires de l'évêque usurier Mousonios de Méloé et celui de Paul d'Olba, qui venait, quant à lui, d'abandonner son siège ${ }^{25}$. Durant la période, Oecuménios continue à recevoir de Sévère un courrier à caractère dogmatique, par exemple une explication sur les idiomes et les opérations, et lui donne l'avis général de ne pas s'en tenir à

19. CConcOec, p. 23-24.

20. Cette section traite en effet des mutilations, du martyre et du suicide.

21. $\quad C L, \mathrm{n}^{\circ} 81, P O 14$, p. $128-131$.

22. PLRE 2, p. 794 : Oecumenius.

23. Breuiarium, p. 133, 21.

24. SL 1, 1, p. 12 [11]. Sévère avait rallié dans la capitale deux évêques isauriens, Serge de Philadelphie et Astérios de Célendéris, et se heurtait à un troisième, Jean de Claudiopolis, qui y défendait les thèses modérément hénoticiennes de Flavien II d'Antioche (SL 1, 1, p. 4 [5] ; Zacharie le Scholastique, Vita Seueri, p. 107). Il réussit à l'isoler de l'épiscopat de sa propre province et des principaux notables locaux. Oecuménios reçut ainsi, dès cette époque, une correspondance théologique de la part de Sévère $\left(C L, \mathrm{n}^{\circ} 1, P O 12\right.$, p. 175-185).

25. SL 1, 23, p. 92 [83]. Cf. Honigmann 1951, p. 86-87, 91, 95 ; Frend 1981, p. $213-$ 215. 
de simples résumés, tandis que la controverse fait rage, pour apprécier le bienfondé de la doctrine sévérienne ${ }^{26}$. L'estime du patriarche pour ce fidèle et qualifié partisan le conduit, d'ailleurs, à envisager avec faveur l'éventualité de l'enrôler dans la cléricature ${ }^{27}$.

À l'extérieur du patriarcat d'Antioche, Sévère trouve un autre confident et un auxiliaire dévoué, autant qu'efficace, en la personne du skholastikos Hippocrate d'Alexandrie ${ }^{28}$, son principal correspondant égyptien. Proche du patriarche Dioscore II (juin 516-14 octobre 517), ce juriste travaille fermement au rapprochement des Églises d'Antioche et d'Alexandrie, pierre angulaire de l'action sévérienne. En termes parfaitement limpides, Sévère insiste auprès de lui, donc de Dioscore, sur la nécessité d'anathématiser expressément le symbole de Chalcédoine (451) et le Tome du pape romain Léon I ${ }^{\text {er }}$ (449) pour réaliser cette union dogmatique ${ }^{29}$. C'est le sens très particulier qu'il donne en effet à l'Hénotique (482), et dont il a convaincu Anastase, pendant son séjour constantinopolitain de 508-511. La communion des principaux patriarcats doit faire la preuve politique de la justesse de cette ligne doctrinale, que le pouvoir impérial peut alors reprendre à son compte. Hippocrate se situe donc au cœur même du dispositif sévérien. Libératus de Carthage présente, quant à lui, le skholastikos Socrate ${ }^{30}$ comme l'inspirateur de l'acceptation de l'Hénotique par Dioscore et de son rejet public du concile de Chalcédoine ${ }^{31}$. Il s'agit assurément là d'une corruption du nom d'Hippocrate, l'agent de Sévère à Alexandrie. L'explication détaillée de sa démarche politico-théologique ne va pas sans confidence personnelle du prélat antiochien. Il expose ainsi à Hippocrate les difficultés qu'il a rencontrées lui-même, de la part du pouvoir et de son délégué, l'a secretis Astérios ${ }^{32}$, après l'envoi à Timothée ${ }^{\mathrm{er}} \mathrm{de}$ Constantinople d'une synodique d'avènement ouvertement antichalcédonienne ${ }^{33}$. Dans la même lettre, en revanche, il s'autorise de Cyrille d'Alexandrie pour

26. $C L, \mathrm{n}^{\circ} 2-3, P O 12$, p. 86-194.

27. $S L 1,5$, p. 38 [35]. Il semble bien que tel fut en effet son destin après 518 : quoique encore discutée, l'identification du personnage avec le commentateur homonyme de l'Apocalypse (CPG 7470) paraît probable ; cf. Dulaey 1982 ; De Groote 1996, p. 91-103 ; LAMOREAUX 1998, p. 100-101.

28. PLRE 2, p. 566 : Hippocrates.

29. $C L, \mathrm{n}^{\circ} 47-48, P O 12$, p. $322-323$.

30. PLRE 2, p. 1019 : Socrates.

31. Breuiarium, p. 133, 2.

32. PLRE 2, p. 172 : Asterius.

33. $C L, \mathrm{n}^{\circ} 46, P O 12$, p. 321. 
justifier, sur le terrain, sa propre prudence en matière de diptyques ${ }^{34}$. Plus loin, il informe Hippocrate des affaires de Cappadoce (diocèse du Pont) et de son refus de la simple convention d'union et de conjonction proposée par le métropolite Sotérichos de Césarée, en deçà du souhait de communion complète exprimé localement par plusieurs évêques ${ }^{35}$. Le contenu d'un tel courrier dépasse clairement la compétence d'un simple particulier, même qualifié. Au-delà d'Hippocrate, c'est bien le patriarche Dioscore d'Alexandrie qui est le véritable destinataire, auprès duquel le skholastikos va jouer le rôle d'un intermédiaire partisan.

D’origine indéterminée ou discutable, deux autres skholastikoi au moins figurent nommément parmi les correspondants de Sévère. Entre 512 et 518, selon l'éditeur moderne, le skholastikos Eusèbe ${ }^{36}$ reçoit du patriarche une explication, de haute teneur philosophique, sur les notions d'essence et d'hypostase ${ }^{37}$. La lecture du fragment conservé laisse supposer qu'il y avait eu discussion préalable et argumentation dans un précédent courrier d'Eusèbe. On doit en déduire, chez l'intéressé, une solide formation intellectuelle, et pas seulement juridique, ce qui situe assez bien le niveau de culture et d'intelligence spéculative atteint, à cette époque, parmi les membres de la corporation. Vers 516-518, Sévère, se référant notamment à Timothée Aelure, expose encore au skholastikos Théophane ${ }^{38}$, comme à Hippocrate, la nécessaire modération qu'il lui faut observer sur la question des diptyques ${ }^{39}$. La ressemblance du propos et la caution d'un autre patriarche alexandrin font supposer que Théophane était lui aussi égyptien. Il se peut, en revanche, que le Zacharie destinataire d'une exégèse de $N b$ 11,16-17 ${ }^{40}$ ne soit autre que le skholastikos homonyme, mentionné précédemment ${ }^{41}$. Établi à Constantinople après sa sortie de l'École de droit (492), il y constituait sans doute, pendant les années du patriarcat, un ferme relais du pontife antiochien.

34. $C L, \mathrm{n}^{\circ} 46, P O 12$, p. 316. Une interprétation radicale de l'anathème de Chalcédoine voudrait que le patriarche exigeât la radiation des diptyques, dans toutes les Églises de son ressort, des noms des évêques défunts signataires au concile de 451, ainsi que de leurs successeurs de même obédience. Sévère préfère laisser la question en suspens, plutôt que d'affronter d'infinies et douloureuses susceptibilités locales. Se prévaloir de Cyrille présente ici le double avantage de se placer dans une perspective antinestorienne incontestable et de souligner l'autorité de l'Église d'Alexandrie.

35. $C L, \mathrm{n}^{\circ} 46, P O 12$, p. $317-319$.

36. PLRE 2, p. 432 : Eusebius 24.

37. $C L, \mathrm{n}^{\circ} 5, P O 12$, p. 195.

38. PLRE 2, p. 1108 : Theophanes 24.

39. $C L, \mathrm{n}^{\circ} 42-43, P O 12$, p. 308-309; $C P G 7071$ (65).

40. $\quad C L, \mathrm{n}^{\circ} 113, P O 14$, p. $277-278$.

41. Cf. n. 7. 
Dans une lettre écrite au métropolite Pierre d'Apamée, son satellite en Syrie $\mathrm{II}^{\mathrm{e}}$, Sévère mentionne avec faveur les noms de quatre autres skholastikoi dont il envisagerait le recrutement dans le clergé ${ }^{42}$. Il recommande ainsi à Pierre les Antiochiens Jean ${ }^{43}$ et Serge ${ }^{44}$, pour l'ordination diaconale. Lui-même consacrerait volontiers les Apaméens Pierre ${ }^{45}$ et Maximin ${ }^{46}$ comme presbytéroi de l'Église d'Antioche. Ces propositions semblent valoir surtout à titre d'hypothèses paradigmatiques, mais elles disent assez l'estime de Sévère et l'influence acquise, dans son esprit, par la corporation. Celles-ci trouvent confirmation dans le traitement que le patriarche propose au métropolite Denys de Tarse (Cilicie $\mathrm{I}^{\mathrm{re}}$ ) et au Magister utriusque militiae per Orientem (MUMPO) Hypatios ${ }^{47}$, qui a connu de l'affaire, pour apurer un contentieux financier opposant à son Église un presbytéros tarsiote dénommé Julien ${ }^{48}$. Sévère envisage la constitution d'une commission ad hoc, composée des évêques de la province assistés de skholastikoi locaux, formés à Béryte, et de deux presbytéroi antiochiens, apparemment leurs confrères, spécialisés dans ces questions. Il s'agirait en somme d'une réunion du synode de Cilicie $\mathrm{I}^{\mathrm{re}}$, élargie à des experts juridiques. Par leur intermédiaire, confiant sans doute dans l'esprit de corps qu'ils manifesteront à son égard, Sévère, lui-même ancien étudiant de Béryte et jurisconsulte, entend affirmer son autorité sur la province. La cohésion disciplinaire et dogmatique qu'il veut réaliser dans son vaste patriarcat s'appuie, en effet, sur un réseau serré de partisans et d'affidés, où les skholastikoi paraissent occuper des positions clefs.

42. SL 1, 5, p. 40-41 [37]. En fait, le propos de cette missive consiste en un point de droit ecclésiastique: l'origine géographique des candidats à la cléricature n'empêche nullement leur incardination dans une autre Église que celle de leur cité de naissance ou de résidence. Sévère entendait couper court à une protestation de Pierre d'Apamée. Le compilateur jacobite ne retient que la règle juridique, quant à lui, et ce fragment ne livre pas le détail du conflit qui opposait les deux évêques, sinon la qualité de skholastikoi que possédaient les personnages pressentis.

43. PLRE 2, p. 608 : Ioannes 61.

44. PLRE 2, p. 994 : Sergius 5.

45. PLRE 2, p. 869 : Petrus 26.

46. PLRE 2, p. 743-744 : Maximinus 14.

47. PLRE 2, p. 577 : Hypatius 6. Neveu de l'empereur Anastase, le MUMPO Hypatios vient prendre le commandement de ses troupes en 517 et Sévère le rencontre à Aigai $\left(\right.$ Cilicie $\mathrm{II}^{\mathrm{e}}$ ), le 25 juillet. C'est vers cette époque que le presbytéros Julien de Tarse, une première fois débouté de ses prétentions, peut avoir abordé un proche d'Hypatios, le curator Héliodore (PLRE 2, p. 532 : Heliodorus 8), puis le MUMPO lui-même.

48. SL 1, 40, p. 126-129 [113-115] ; 5, 5, p. 329 [293-294]. 


\section{Analyse}

À ce stade, il convient de préciser la nature des fonctions que le patriarche d'Antioche semble attribuer de préférence à ces skholastikoi. Remarquons tout d'abord qu'ils se trouvent assez souvent investis de missions de confiance, à caractère généralement informel. À Antioche, on voit ainsi le skholastikos Jean porter une lettre personnelle, de la part de Sévère, à l'évêque suffragant Antonin de Béroia ${ }^{49}$. Peut-être le message se doublait-il d'un commentaire oral, dont la teneur nous échappe, mais qu'autorisent à envisager les étroites relations qui liaient Sévère et Antonin ${ }^{50}$. On sait qu'en Isaurie Oecuménios jouit de la confiance totale du patriarche, qui en a fait son représentant pour les affaires disciplinaires enchevêtrées que connaît la province ${ }^{51}$, face à un métropolite (Solon de Séleucie) demeuré jaloux de ses prérogatives ${ }^{52}$. C'est l'amitié de Sévère qui préside à ce choix, autant que les compétences techniques d'Oecuménios, mais celui-ci ne paraît tenir aucun rang dans l'« organigramme» de l'Église locale, non plus que dans celle d'Antioche. Le responsable en titre de la discipline des évêques isauriens reste, en principe, l'archidiacre de Séleucie ${ }^{53}$, un dénommé Calliste, luimême compromis ${ }^{54}$, tandis que Solon défend bec et ongles l'autorité du synode provincial. Oecuménios ne peut donc intervenir qu'à titre de conseiller dont les avis ne possèdent pas de force exécutoire, tant que les affaires en cours n'auront pas été portées devant la juridiction patriarcale. D'autre part, on sait que Sévère ne l'a pas ordonné parmi les clercs d'Antioche. De même, à Alexandrie, le rôle politique du skholastikos Hippocrate, assurément capital, n'a rien d'officiel non plus. Hippocrate n'est pas l'apocrisiaire de Sévère auprès de Dioscore II, mais un agent d'influence, présent dans l'entourage du patriarche égyptien. À Constantinople, on peut légitimement supposer que la vieille amitié de Zacharie le skholastikos prête son aide à Sévère tant à la cour impériale qu'auprès de Timothée II, mais on connaît, d'autre part, le véritable apocrisiaire d'Antioche, qui n'est autre que

49. $S L 1,16$, p. 68 [61].

50. Honigmann 1951, p. 25-26; PLRE 2, p. 107 : Antoninus 1.

51. FREND 1981, p. 211-215.

52. AlPI 2002, p. 534-535.

53. BréHIER 1970, p. 410. Par délégation de l'évêque, l'archidiacre veille en effet à la discipline des clercs, tel un moderne « vicaire général ». Dans une métropole, cette autorité s'étend aux évêques suffragants, au moins dans les vacances du synode provincial.

54. Intransigeant sur le chapitre des diptyques, au rebours de la modération préconisée par Sévère, Calliste se trouve bientôt convaincu de simonie et de faux en écriture. HoNIGMANN 1951, p. 85-87 ; FREND 1981, p. 212-214. 
le propre frère du patriarche, le presbytéros Pierre ${ }^{55}$. Sévère use donc volontiers, dans le cadre de missions ponctuelles ou spécifiques, mais en marge des canaux ordinaires du concours officieux de membres d'une corporation qu'il connaît bien.

L'affaire du presbytéros Julien de Tarse nous rapporte directement à la fonction première et à la spécialité originelle des skholastikoi. Il s'agit en effet de démêler un contentieux juridico-financier. Les professionnels locaux, des laïcs de formation bérytaine, et leurs confrères ecclésiastiques, dépêchés par Sévère depuis Antioche, se trouvent ici dans leur rôle ordinaire. L'épisode n'en est que plus révélateur de l'ascendant qu'ils exercent, puisqu'ils vont dominer une commission qui constitue en fait une réunion synodale, et donc prendre le pas sur les évêques ciliciens. Il y a urgence pour Sévère, car il faut détourner Julien de se pourvoir devant la justice civile, comme il a tenté de le faire auprès du MUMPO Hypatios. On mesure ici le rôle dévolu aux skholastikoi dans le renforcement des institutions ecclésiastiques, avec lequel se confond alors la défense de la théologie sévérienne. Telle qu'elle nous est conservée, la correspondance du patriarche révèle, d'ailleurs, plusieurs contentieux du même ordre, où se trouvent impliqués des évêques : en Cilicie $\mathrm{II}^{\mathrm{e}}$, les sous-diacres d'Alexandrette en conflit avec Jean ${ }^{56}$, ou encore les clercs de Flavias en conflit avec Procope ${ }^{57}$; en Pamphylie, les sousdiacres de Pergé que Castor ne défend pas des torts qu'ils subissent de la part d'autres clercs ${ }^{58}$. Partout on sollicite l'arbitrage de Sévère, ce qui implique un examen juridique, au cas par cas, et donc le concours de spécialistes, pour préparer les dossiers et éclairer le patriarche. De même que celui-ci, en matière théologique, doit adresser continuellement des formules de satisfaction, fondées sur son interprétation des Écritures et de la tradition patristique, pour régler localement des dissidences doctrinales ${ }^{59}$, de même sa chancellerie ne cesse-t-elle de rédiger des attendus disciplinaires, en conformité avec les canons ecclésiastiques ${ }^{60}$. On

55. SL 1, 49, p. 149-150 [134-135] ; 11, p. 518 [461] ; Honigmann 1951, p. 22. Par ailleurs, Sévère montre l'importance qu'il attache aux fonctions statutaires d'apocrisiaire, sans préciser toutefois les noms des titulaires, cf. SL 1, 17 p. 72 [65] ; 47, p. 144 [130] ; 48, p. 145-146 [131] ; 4, 7, p. 299 [266].

56. SL 1, 32, p. 110-111 [98-99].

57. $S L 1,13$, p. $59-60$ [53-54].

58. SL 1, 7, p. 43-45 [39-41]. Pergé appartient au diocèse d'Asie, mais Castor est entré dans la communion de Dioscore et de Sévère, sans doute avec son clergé. Faute de pouvoir saisir le synode pamphylien, les sous-diacres se pourvoient donc devant la juridiction du patriarche d'Antioche. Cf. Honigmann 1951, p. 132 ; PChBE 3, p. 573-774 : Kastôr 1.

59. SL 5, 3, p. 320 [285] ; 4, p. 324 [289] ; 5, p. 326 [291].

60. Honigmann 1961, p. 50-51. 
devine la présence d'une cellule juridique antiochienne fort active, à laquelle correspondent, dans les métropoles des provinces, des services analogues, comme on le voit à Bosra ou à Tarse. Les skholastikoi se trouvent là en première ligne. On doit supposer aussi tout un travail de bureau, un personnel de notarii et de copistes, le même, sans doute, que celui que Sévère mentionne à propos des formulaires sur la foi, souvent «standardisés » ${ }^{61}$. Dans une lettre à Antonin de Béroia sur la licéité d'une ordination extraterritoriale, courrier auquel il aurait voulu joindre un exemplaire type de jugement rendu, le patriarche se plaint ainsi de manquer de préposés aux écritures ${ }^{62}$. Ajoutons que cette importance avérée des chancelleries des évêques et du patriarche, cette bureaucratie ecclésiastique, où dominent les skholastikoi, concordent avec ce que peut nous apprendre l'archéologie des groupes épiscopaux ${ }^{63}$.

Expertise technique et montée en influence se rejoignent, bien sûr: la complexité des procédures, la nécessité où se trouve Sévère de maintenir la cohésion disciplinaire, autant que la communion doctrinale, dans son vaste et contrasté ressort, lui rendent les skholastikoi d'autant plus nécessaires et précieux. C'est donc volontiers parmi eux qu'il choisira aussi des auxiliaires et des agents, même pour des tâches qui débordent apparemment le strict domaine juridique, ainsi Jean d'Antioche ou Hippocrate d'Alexandrie. La solidarité de corps a pu jouer, en la matière, et la sympathie d'une même formation intellectuelle, voire la camaraderie naturelle entre anciens élèves de l'École de Béryte. Le cas semble évident pour Zacharie, qui mène une active campagne d'opinion, depuis Constantinople, en diffusant sa Vita Seueri. Il reste que la présence d'assesseurs juridiques permanents dans l'entourage des évêques orientaux apparaît bien comme un trait caractéristique de l'époque ${ }^{64}$. Sévère se trouve déjà dans la position de son successeur chalcédonien Grégoire (570-593), dont on connaît deux célèbres conseillers skholastikoi, les historiens Jean et Évagre d'Épiphanie ${ }^{65}$. Après Sévère, signe de l'ascendant acquis par la corporation, deux skholastikoi orientaux vont encore accéder à la dignité de patriarche : à Antioche, Anastase (559-570/593-598) ; à Constantinople, l'Antiochien Jean III (565-577). Peu à peu s'est instaurée une

61. SL 5, 3, p. 320 [285]; 4, p. 324 [289].

62. SL 1, 14, p. 65-66 [59].

63. Les vestiges du groupe épiscopal de Résafa (Euphratésie méridionale), datables du début du vi ${ }^{\mathrm{e}}$ siècle, comportent ainsi une pièce destinée au travail de la chancellerie épiscopale, adossée au chevet de la basilique de la Sainte-Croix (= «basilique A ») et définie par le fouilleur comme «Scriptorium oder Archiv ». Cf. UlberT 1986, p. 146. Il semble préférable de voir dans ce local un scrinium dont l'agencement reste à étudier.

64. Lamoreaux 1995, p. 160.

65. Pour la composition de leurs œuvres, ils ont pu bénéficier, l'un et l'autre, d'un accès d'office aux archives patriarcales. Cf. Allen 1981, p. 10-11. 
interpénétration des milieux ecclésiastique et juridique, qui semble culminer à la fin du siècle et dont témoigne déjà la correspondance sévérienne. On a pu avancer, d'ailleurs, que l'enseignement dispensé à Béryte a préparé en quelque sorte cette symbiose au plan intellectuel ${ }^{66}$. De fait, la communion théologique que promeut Sévère, tant entre les Églises du ressort d'Antioche qu'avec celles d'Alexandrie ou de Constantinople, comme lien juridico-sacramentel enlaçant les membres de la véritable Église du Christ, à l'exclusion des adversaires dogmatiques ${ }^{67}$, porte bien la marque d'une ecclésiologie de type résolument contractuel, dans l'esprit même du droit romain. La question des diptyques, débattue avec Hippocrate ou Théophane, l'illustre particulièrement. Il paraît donc tout à fait logique que le même personnel s'inquiète à la fois de droit et de dogme, que des skholastikoi, tels Jean de Bosra, Eusèbe ou Oecuménios, montrent une réelle curiosité et culture théologiques, que les mêmes locaux de la chancellerie patriarcale abritent la rédaction des règlements de contentieux et des formulaires de foi, indéfiniment reproduits par les mêmes copistes.

Un point particulier éclairerait ce rôle d' « intellectuels organiques » que jouent les skholastikoi auprès du patriarche d'Antioche. Il s'agit peut-être d'une spécificité syrienne, au moins à l'origine. On sait que Jean le skholastikos, patriarche de Constantinople, s'est illustré par son souci de rassembler les canons ecclésiastiques, dont il a composé le premier recueil systématique conservé jusqu'à nous, la Synagôgè kanonôn. Or, il se serait fondé, pour ce faire, sur un ouvrage anonyme du milieu du $\mathrm{VI}^{\mathrm{e}} \mathrm{s}$. qui réunissait sous soixante titres une collection antérieure, et auquel il aurait eu accès à Antioche, tandis qu'il y exerçait encore comme jurisconsulte ${ }^{68}$. Tel qu'on a pu le reconstituer à partir de sa correspondance, le kanonikon de Sévère marquerait, quant à lui, une étape encore plus ancienne, et précéderait de peu celui dont disposait le patriarche Éphrem (527-545), sans doute la source consultée par Jean ${ }^{69}$. On entrevoit donc un long travail de compilation juridique, dont la chancellerie d'Antioche serait la matrice, et ses skholastikoi les maîtres d'œuvre, traduction théorique de l'expérience juridictionnelle variée du siège patriarcal. Sévère fut peut-être l'initiateur d'une telle entreprise, qui procédait du même esprit que la composition du Code de Justinien, en droit civil, et qui lui était à peu près contemporaine. Dans l'un et l'autre cas, ce sont les méthodes élaborées par les maîtres æecuméniques de l'École de Béryte qui auront été appliquées. L'estime dans laquelle le patriarche tenait cet établissement et ses

66. McNAMEE 1998, p. 288, décrit une «symbiosis between church and law school in late antiquity ». Pour cet auteur, la pratique pédagogique des maîtres bérytains serait à l'origine aussi bien des commentaires juridiques marginaux que des chaînes exégétiques ou des gloses littéraires.

67. DE VRIES 1957, p. 116.

68. BRÉHIER 1970, p. 356 ; VAN DER WAL - LOKIN 1985, p. 51-54.

69. Honigmann 1961, p. 50-51, 82-83. 
indispensables alumni, exprimée notamment dans sa lettre au MUMPO Hypatios à propos de Julien de Tarse ${ }^{70}$, se comprend d'autant mieux.

Il ne résulte pas de cette proximité du patriarche et des skholastikoi que ceux-ci soient normalement des clercs. La correspondance de Sévère ne signale une telle qualité que dans le seul cas des deux presbytéroi anonymes de l'affaire Julien, dépêchés d'Antioche à Tarse ${ }^{71}$. Leur compétence technique ne se trouve pas formellement désignée, mais elle se déduit du contexte, et du parallèle établi par l'épistolier avec les skholastikoi de Cilicie que viennent renforcer ces deux ecclésiastiques. On peut ainsi considérer que tout personnage qualifié par Sévère de skholastikos est un laïc au moment de la rédaction de la lettre qu'il reçoit ou qui le mentionne. Si tel professionnel entrait dans les ordres, son nouvel état imposerait désormais, à son propos, l'appellation de très pieux ou très dévot, suivie de l'énoncé de son grade dans la cléricature. Inversement, quand le patriarche évoque l'heureuse (mais improbable) éventualité de consacrer lui-même les skholastikoi Oecuménios, Pierre et Maximin, ou de voir ordonner Jean et Serge en Syrie II ${ }^{\mathrm{e}}$, c'est bien que les intéressés ne sont pas des clercs au moment de ce vœu. Zacharie obtiendra certes l'épiscopat, mais seulement après 518, et au sein de l'Église chalcédonienne ${ }^{72}$. Le commentaire de l'Apocalypse d'Oecuménios peut suggérer que son auteur avait également embrassé l'état ecclésiastique, mais la tradition grecque de cette exégèse paraît indiquer qu'il se situait alors, lui aussi, dans le camp de l'Église impériale et orthodoxe, soit après l'éviction de Sévère ${ }^{73}$. De manière récurrente, d'ailleurs, ce dernier se plaint amèrement, dans sa correspondance, de la déplorable situation de sa trésorerie, avouant sans fard que cette détresse financière le conduit justement à limiter les ordinations ${ }^{74}$. Celles de skholastikoi sont donc exceptionnelles. Comme assesseurs juridiques, ils doivent assurément

70. SL 1, 40, p. 127 [114].

71. SL 1, 40, p. 127 [114].

72. Il signe contre Sévère en 536, au synode de Constantinople, comme titulaire du siège de Mytilène $(C P G 6930=9331$ [1]). La promotion à l'épiscopat de ce skholastikos n'en illustre pas moins la montée en puissance de sa corporation d'origine.

73. Les textes antichalcédoniens ont été bannis de la tradition grecque, interdits de copie et de reproduction, à commencer par les écrits de Sévère lui-même, dès 536. Ils n'ont essentiellement subsisté qu'en traduction syriaque, réalisée par les soins des dissidents jacobites. Il en va pareillement de la Vita Seueri de Zacharie de Mytilène, tandis que la partie de l'œuvre de cet auteur qui se trouve postérieure à son ralliement à l'orthodoxie de l'Église impériale a été conservée en grec.

74. SL 1, 8, p. 45-48 [41-44] ; 17, p. 70-73 [63-66]. Tout clerc perçoit une rétribution annuelle, proportionnelle à son grade, prise sur les revenus de l'Église d'Antioche ou de tel sanctuaire ou fondation particulière à laquelle il se trouve attaché. La législation impériale tend donc à limiter le nombre des ordinations en fonction du revenu effectivement disponible. 
recevoir une rémunération de l'Église, pour chaque dossier traité, sans préjudice des revenus qu'ils peuvent toucher par ailleurs, mais il ne s'agit pas de rétribution cléricale. Certains travaillent peut-être aussi, ou ont travaillé, pour quelque service de l'État, et c'est sans doute la raison du titre que porte le comte Oecuménios.

Ainsi, entre 512 et 518, la correspondance officielle de Sévère d'Antioche nous présente un dossier assez bien circonstancié sur une douzaine de skholastikoi. Celui-ci permet de mesurer l'influence acquise par une corporation devenue nécessaire au fonctionnement régulier de la juridiction ecclésiastique, au moment où le patriarche lutte de vive force pour assurer la cohésion, disciplinaire et doctrinale, du diocèse oriental. D'assesseurs juridiques attitrés, ces professionnels du droit deviennent ainsi les agents, dans la pratique, d'une politique et d'une théologie volontaristes. Un tel glissement s'explique dans le contexte culturel de l'époque et de la région, qui voit le droit déterminer aussi bien les formes du gouvernement que les modes de pensée. L'influence intellectuelle et technique de l'École de Béryte s'affirme d'autant mieux qu'elle rencontre ici la pleine adhésion de Sévère, soucieux de porter la législation canonique à un niveau de rationalité comparable à celui du droit civil. L'interpénétration entre les milieux juridique et ecclésiastique, entre le dogme et le droit, annonce la symbiose que l'on pourra constater vers la fin $\mathrm{du} \mathrm{VI}^{\mathrm{e}} \mathrm{s}$. et qui va caractériser le monde byzantin.

Frédéric AlPI

\section{Abréviations}

Les abréviations des autres périodiques suivent l'usage de L'Année philologique.

ACO = Acta conciliorum oecumenicorum, éd. E. Schwartz et al., Strasbourg, Leipzig, Berlin (1914- ).

CConcOec $=$ Discipline générale antique, 1, 1, Les canons des conciles æecuméniques [voir Textes anciens].

$C L=A$ collection of letters of Severus of Antioch .

$C P G=$ M. Geerard et al., Clauis Patrum Graecorum, Turnhout (1979-1998, 2003).

$C P L=$ E. Dekkers - A. Gaar, Clauis Patrum Latinorum, Steenbrugge ${ }^{3}$ (1995).

DSp = Dictionnaire de spiritualité : ascétique et mystique, doctrine et histoire, éd. M. Viller et al., Paris (1933-1995).

OSyr = L'Orient syrien : revue trimestrielle d'études et de recherches sur les Églises de langue syriaque, Paris (1956-1967).

PChBE = Prosopographie chrétienne du Bas-Empire, 3, Prosopographie du diocèse d'Asie (325-641), S. Destephen, Paris (2008).

$P O=$ Patrologia orientalis, Paris, puis Turnhout (1903-). 
PLRE 2 = The prosopography of the later Roman Empire, 2, 395-527, by J. R. Martindale,

Cambridge (1980).

$R E=$ Realencyklopädie des classischen Altertumswissenschaft, Munich (1914-1980).

$S L=$ The sixth book of the select letters of Severus, patriarch of Antioch.

\section{Textes anciens}

Discipline générale antique. 1, 1, Les canons des conciles æecuméniques (II [sic]-IXe s.) [CConcOec], éd. et trad. fr. P.-P. Joannou, Grottaferrata (1962-1963) [CPG .8513].

LibÉRAtus DE CARThage, Breuiarium causae Nestorianorum et Eutychianorum, éd. E. Schwartz, dans : ACO 2/5 (1936), p. 98-141 [CPL 865].

SÉvÈre D'AnTIOChe, The sixth book of the select letters of Severus Patriarch of Antioch in the Syriac version of Athanasius of Nisibis, éd. et trad. angl. E. W. Brooks, Londres (1902-1904) [CPG 7070 (1)].

SÉvÈre D'ANTioche, A collection of letters of Severus of Antioch, éd. et trad. angl. E.W. Brooks, dans : PO 12/2 (1915), p. 163-342; 14/1 (1920), p. 1-310 [CPG 7070 (2)].

Socrate de Constantinople, Histoire ecclésiastique. Livre 1, éd. G. Ch. Hansen, trad. fr. P. Périchon et P. Maraval, introd. et notes par P. Maraval, Paris (2004) (Sources chrétiennes 477) [CPG 66028].

Zacharie le Scholastique, Vita Seueri, éd. et trad. fr. M.-A. Kugener, dans : PO 2/1 (1904), p. 3-115 [CPG 6999].

\section{Études modernes}

AlLEN 1981

P. Allen, Evagrius Scholasticus, the church historian, Louvain.

ALPI 2002

Fr. ALPI, Recherches sur l'administration et la pastorale de Sévère d'Antioche (512-

518), thèse de doctorat de l'Université de Paris IV.

BRÉHIER 1949

L. BRÉHIER, Les institutions de l'empire byzantin, Paris ( $\left.{ }^{2} 1970\right)$.

Claus 1965

A. Claus, $O \sigma \chi O \lambda \alpha \sigma \tau \imath \kappa o ́ \varsigma$, Köln.

Collinet 1925

P. Collinet, Histoire de l'école de droit de Beyrouth, Paris.

De Groote 1996

M. De Groote, « Die Quaestio Oecumeniana », SEJG 36, p. 67-105.

Dulaey 1982

M. Dulaey, « Oecuménius », $D S p$ 11, col. 681-682. 


\section{FREND 1981}

W.H.C. Frend, «Isauria: Severus of Antioch's problem-child, 512-518», in

F. PASchKe (éd.), Überlieferungsgeschichtliche Untersuchungen, Berlin, p. 209-216.

HonigmanN 1951

E. Honigmann, Évêques et évêchés monophysites d'Asie antérieure au vie siècle, Louvain.

\section{HONIGMANN 1961}

E. Honigmann, « Le concile de Constantinople de 394 et les auteurs du Syntagma des XIV titres », in Id., Trois mémoires posthumes d'histoire et de géographie de l'Orient chrétien, Bruxelles, p. 1-83.

LAMOREAUX 1995

J.C. Lamoreaux, « Episcopal courts in Late Antiquity », JECS 3, p. 143-167.

LAMOREAUX 1998

J.C. Lamoreaux, « The provenance of Ecumenius' Commentary on the Apocalypse », VChr 52, p. 88-108.

LANIADO 2002

A. LANIADO, Recherches sur les notables municipaux dans l'empire proto-byzantin, Paris.

Marrou 1948

H.-I. Marrou, Histoire de l'éducation dans l'Antiquité, Paris ( $\left.{ }^{6} 1965\right)$.

MCNAMEE 1998

K. McNamee, « Another chapter in the history of scholia », $C Q 48$, p. 269-288.

PreISIGKE 1921

F. Preisigke, RE $2 \mathrm{~A} / 1$, s. v. «'O $\sigma \chi 0 \lambda \alpha \sigma \tau 1 \kappa o ́ \varsigma »$, col. 624-625.

ULBERT 1986

Th. Ulbert, Resafa. 2, Die Basilika des heiligen Kreuzes in Resafa-Sergiupolis, Mayence.

DE VRIES 1957

W. de VRIES, « La conception de l'Église chez les Syriens séparés de Rome », OSyr 2, p. 111-124.

VAN DER WAL - LOKIN 1985

N. VAN DER WAL - J.H.A. LoKIN, Historiae iuris graeco-romani delineatio : les sources du droit byzantin de 300 à 1453, Groningen. 